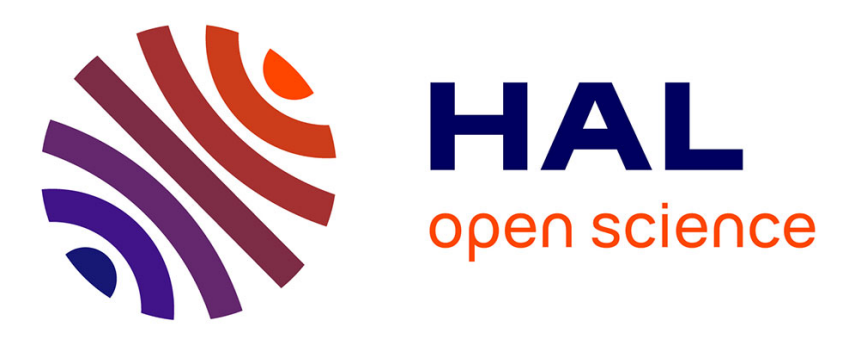

\title{
Jean José Marchand et les Archives du XXe siècle Yves Chevrefils Desbiolles
}

\section{To cite this version:}

Yves Chevrefils Desbiolles. Jean José Marchand et les Archives du XXe siècle. Valentine Robert, Laurent Le Forestier, François Albera. Le Film sur l'art. Entre histoire de l'art et documentaire de création, Presses universitaires de Rennes, pp.261-271, 2015. hal-03164816

\section{HAL Id: hal-03164816 \\ https://hal-normandie-univ.archives-ouvertes.fr/hal-03164816}

Submitted on 10 Mar 2021

HAL is a multi-disciplinary open access archive for the deposit and dissemination of scientific research documents, whether they are published or not. The documents may come from teaching and research institutions in France or abroad, or from public or private research centers.
L'archive ouverte pluridisciplinaire HAL, est destinée au dépôt et à la diffusion de documents scientifiques de niveau recherche, publiés ou non, émanant des établissements d'enseignement et de recherche français ou étrangers, des laboratoires publics ou privés. 


\title{
Jean José Marchand et les Archives $d u X X^{e}$ siècle
}

\author{
Yves Chevrefils Desbiolles \\ Institut Mémoires de l'édition contemporaine (IMEC)
}

Critique d'art, de cinéma et de littérature, Jean José Marchand est né le 14 août 1920 à Paris. Il est décédé dans la même ville le 8 mars 2011. Rejeton d'une famille aux racines bretonnes, ses parents sont issus de ces deux solitudes qui ont façonné la France : côté paternel, on est franc-maçon et positiviste ; côté maternel, on est catholique pratiquant. Mais la simplicité de ce schéma ne répond pas toujours à la réalité. Jean José Marchand en donne un exemple dans un long entretien filmé chez lui en octobre $2005^{1}$ : son grand-père maternel refuse de suivre les Communards en 1870 , mais est scandalisé par la violence extrême de la répression qui a suivi la prise de Paris, violence qui aurait surtout été le fait des paysans engagés dans les troupes versaillaises et de la haine meurtrière éprouvée par ceux-ci envers les ouvriers. Ces remarques biographiques - pour anecdotiques qu'elles soient - mettent en perspective la courbe de vie de Jean José Marchand caractérisée par des inflexions singulières, un peu à l'image de ce père officier dans l'armée coloniale et de sa mère qui, à la maison, montre de belles dispositions pour le dessin.

Écolier, Jean José Marchand est bagarreur : «La récréation était le lieu de la violence », dit-il, mais « la maison, c'était le lieu de la lecture ». Au lycée Voltaire, au moment où sous le Front populaire un antisémitisme s'exprime de plus en plus ouvertement dans certains milieux politiques et intellectuels français, ses amis sont le plus souvent juifs « parce que je sentais bien que les Juifs [dit-il, étaient] des marginaux, dans notre société [...], et moi, j'étais un marginal. » Ces mêmes années troubles persuadent pourtant le jeune Jean José Marchand que la culture littéraire et artistique peut et doit « réconcilier le peuple entier » avec lui-même, utopie vite refroidie par le désenchantement de la guerre.

Engagé volontaire dans un régiment alpin durant la guerre, ils ne sont que trois à l'intérieur de son unité, en juin 1940, à exprimer ouvertement leur opposition à l'armistice signé par le maréchal Pétain : un sympathisant de l'Action française, un communiste et lui. Jean José Marchand souhaite vite rejoindre les Forces françaises

\footnotetext{
${ }^{1}$ Entretien avec Guillaume Louet filmé par Benoît Bourreau du 24 au 28 octobre 2005, produit par l'Institut national de l'audiovisuel (INA). Des extraits de cet entretien sont repris dans le documentaire intitulé Jean José Marchand. Mieux partagés que nous ne sommes, produit par le Studio national des arts contemporains Le Fresnoy en 2006. Je tiens ici à remercier pour leurs conseils et leur aide Guillaume Louet, Claire Paulhan, Élisabeth Kapnist et Annie Chevrefils Desbiolles.
} 
libres, mais rate son départ pour Londres (voyageur qui restera toute sa vie distrait, il se présente trop tardivement au point de rendez-vous clandestin d'où devait partir un avion). Il se rapproche alors des milieux intellectuels résistants, d'abord celui gravitant autour des Cahiers du Témoignage chrétien. Il devient à l'occasion porteur de tracts. Réfugié à Marseille, il fréquente beaucoup Les Cahiers $d u$ Sud et fait la connaissance de Simone Weil. Avec le poète André Verdet, il distribue Défense de la France. Il enseigne brièvement la littérature dans un lycée d'Aix-en-Provence avant de remonter à Paris vers la fin de 1942 pour occuper un poste de bibliographe chargé de l'inventaire des revues à la Bibliothèque nationale de France. Les revues deviennent à la fois son gagne-pain et le lieu qu'il habite. Il collabore à Poésie 41 et à Poésie 42, à Toutes Aures, aux Cahiers du sud, à Confluences. À la demande de René Tavernier, directeur de Confluences, il travaille intensément à une anthologie de la poésie française du premier $\mathrm{XX}^{\mathrm{e}}$ siècle. Le projet, pourtant fort avancé, n'aboutit pas, mais permet à Jean José Marchand de prendre conscience d'une partie de lui-même : il voue un culte au détail, à la précision, à la rigueur des mots, des choses, des hommes. Les années de guerre sont pour Jean José Marchand celles où il fait la connaissance de « tout le monde », moins au travers de la vie mondaine ambiguë de ce temps que grâce aux milieux qu'il fréquente, proches de la poésie et de la littérature, grâce aussi au cinéma, qu'il fréquente beaucoup comme un grand nombre de Français de ce temps.

\section{Le journalisme, la radio, la sociologie}

Tout ce qui s'est discrètement mis en place durant l'occupation prend forme à la Libération : Jean José Marchand écrit des textes pour une chronique radiophonique, «Paris vous parle »; il est critique de cinéma à Volonté de Ceux de la Résistance, puis à Climats ; il est critique littéraire au Magasin du spectacle ; il est journaliste à FrancTireur. En 1946, Jean José Marchand entre à Combat en tant que critique d'art pour, semble-t-il, répondre à une un inquiétude d'Albert Camus qui souhaitait équilibrer la chronique régulière de Charles Estienne, tournée de manière trop exclusive vers le surréalisme et l'abstraction. Sa mission : faire le tour des expositions. Il le fera jusqu'en 1948, année du départ de Camus à la tête de Combat. Il le fera aussi pour Paru après que Claude Bourdet eut mis fin à ses fonctions à Combat.

En art, l'approche de Jean José Marchand aura été résolument critique, mais ouverte à tous les tendances pratiquées par la jeune peinture de ce moment, dominée par l'expressionnisme et par l'abstraction : lorsque il reprochait à certains jeunes peintres leur recours à un contenu sentimental ou théologique, Jean José Marchand s'adressait d'abord - comme il le dira beaucoup plus tard à Guillaume Louet - aux peintres communistes, André Fougeron en tête ; il aimait Bram Van Velde et Asger Jorn, mais déplorait pour tous les deux ce qu'il analysait comme « un manque de travail », c'est-à- 
dire probablement un goût excessif pour l'inachevé ; il s'intéressait également à Picabia et Kandinsky, mais qui à vrai dire restaient à ses yeux, «des littérateurs » plus que « de purs peintres ». Sa plus grande admiration allait à Hans Hartung ${ }^{2}$ et à Mathieu, peintres qu'il considérait comme « essentiels » à la compréhension de l'art issu de l'aprèsguerre. Jean José Marchand est d'ailleurs le premier, dans le catalogue d'une exposition - L'Imaginaire en 1947 - conçue par Mathieu à la Galerie du Luxembourg, à parler d'abstraction lyrique dans sa variante syntagmatique d'abstractivisme lyrique . $^{3}$. Jean José Marchand le dira beaucoup d'années plus tard à Guillaume Louet, toute sa vie aura été marquée par une attirance paradoxale pour l'ordre et pour la révolte, toute sa pensée politique restera portée par une certaine idée de la France fondée sur son autonomie au sein du concert des nations, animée par une méfiance envers le parlementarisme, mais aussi par un désir de justice dans les rapports entre les classes sociales. Où pourra-t-il réaliser un idéal en apparence aussi contradictoire ? Jean José Marchand ne se reconnaît bien sûr ni dans les régimes de type autoritaires qu'il a combattus, ni dans les variantes du marxisme auquel il ne croit pas. Le gaullisme naissant - sa méfiance envers les rhétoriques parlementaires, son goût pour une économie encadrée par un État fort - deviendra alors le lieu politique naturel de Jean José Machand dès la fondation du Rassemblement du peuple français (RPF) par le général en avril 1947. Cet engagement militant se poursuivra jusqu'en 1954. Jean José Marchand travaille alors avec Pascal Pia à l'Agence Express, l'agence de presse du RPF. Sans surprise, on le retrouve aussi critique littéraire pour Le Rassemblement, l'organe du même RPF. Il fait la connaissance d'André Malraux qu'il admire tant pour ses idées que pour le pragmatisme avec lequel il les réalisait.

Cette longue période d'engagement politique et de journalisme, cette profusion de contributions littéraires et artistiques révèlent un tempérament hyperactif et idéaliste. Mais il témoigne aussi d'un jeune homme aux prises avec les nécessités alimentaires du moment. Pour gagner sa vie, il entre au mitan des années 1950 au service de l'Institut de sociologie grâce à Raymond Aron qui le présente à un de ses directeurs, Daniel Lerner. Ce dernier dirige alors des travaux sur les usages, les pratiques et les normes en vigueur au sein de la classe dirigeante française. Puis, dans le cadre du jeune CNRS avec Michel Crozier, Alain Touraine et d'autres, il participe à différentes enquêtes dont celle sur les

\footnotetext{
2 Jean José Marchands, Vie aux frontières du poème. Essais de prospection poétique. Avec un dessin de Hans Hartung, Paris, Minuit, 1955 ; réédition Genève, Slatkine, 1982, augmentée de quelques poèmes.

${ }^{3}$ Voir Mathieu. 50 ans de création, Hervas, 2003.
} 
employés en France ${ }^{4}$. Dès cette époque, Jean José Marchand s'exerce avec succès à l'art de l'interview.

En 1958, c'est le grand retour à la radio avant de rapidement passer à la télévision, cela grâce bien entendu à ses talents et à son expérience, mais aussi - il faut le dire - grâce à ses affinités gaullistes. Dans son entretien avec Guillaume Louet, Jean José Marchand ne cache pas que la télévision française était bien à cette époque une télévision d'État au sens immédiat du terme, c'est-à-dire au service du pouvoir à la tête de cet État ${ }^{5}$. Jean José Marchand entre donc à la RTF (Radio-Télévision française) en tant que «protégé » d'Albert Ollivier, ancien résistant, ex-directeur du journal gaulliste Le Rassemblement, lui-même un «protégé » d'André Malraux. Jean José Marchand est intégré au secrétariat général de la Direction - c'est-à-dire, à l'époque dense de la Guerre d'Algérie, chargé de la préparation des dossiers et des courriers - avant d'être en 1959 propulsé à la tête du service Cinéma, responsable du choix des films et des séries et de la production de feuilletons ${ }^{6}$. Il n'hésite pas dès cette époque à programmer le dimanche soir, à l'heure de la plus grande écoute, des films considérés comme difficiles, ce qui lui vaut moult récriminations de la part du public. Près de dix ans plus tard, après les événements de Mai 68 qui ont pourtant fortement déstabilisé le pouvoir du général de Gaulle, c'est encore à sa fidélité gaulliste que Jean José Marchand doit de rester chef du même service Cinéma (délesté, il est vrai, de sa mission de production de feuilletons français ).

\section{Apollinaire d'abord}

Libéré des feuilletons, Jean José Marchand voudrait bien se tourner vers le film documentaire. Depuis longtemps, il souhaitait interviewer celui pour qui il éprouvait la plus profonde admiration littéraire : Jorge Luis Borgès. Son projet se heurte au refus du Directeur de ce qui s'appelle dorénavant l'ORTF (Office de radiodiffusion et télévision française) après le décès prématurée d'Albert Ollivier. Cette impatience à entrer de plain-pied dans la production de films documentaires trouve son apaisement lorsque,

\footnotetext{
${ }^{4}$ Voir Michel Crozier, Le Monde des employés de bureau. Résultats d'une enquête, menée dans sept compagnies d'assurances parisiennes, Paris, Seuil, 1964. Voir aussi La Querelle de la C.E.D. Essais d'analyse sociologique. Recueil d'études par Jacques Fauvet, Alfred Grosser, Stanley Hoffmann, Jean José Marchand, Jean Stotzel...., sous la direction de Raymond Aron et Daniel Lerner, Paris, Librairie Armand Collin, 1956.

${ }^{5}$ Cette mainmise politique sur la télédiffusion constitue le grand procès historique fait à l'ORTF, qui mènera à son éclatement en 1974.

${ }^{6}$ Jean José Marchand, « Un cabinet de curiosité aux portes de Paris ». Entretien avec Eve-Marie Cloquet, Le Journal des lettres et de l'audiovisuel, 1996.
} 
dans la grande maison ORTF, on se souvient soudain en 1968 que cinquante ans plus tôt, le 13 novembre 1918, à Paris à la veille de l'Armistice, Guillaume Apollinaire était mené en terre par un tout petit groupe d'amis. L'ORTF décide de souligner l'événement et demande à son chef du service cinéma de concevoir une émission. La première question que se pose alors Jean José Marchand est la suivante : cinquante plus tard reste-t-il des témoins des derniers jours de vie d'Apollinaire et de cette réunion si particulière au cimetière du Père Lachaise ? Une enquête rapide révèle qu'un petit nombre d'octogénaires et de nonagénaires un peu oubliés de tous sont toujours de ce monde. Leurs noms ? André Salmon, André Billy, Roland Dorgelès, Georges Ribemont-Dessaignes ou Gabrielle Buffet Picabia ${ }^{7}$. Pour cette émission intitulée Apollinaire cinquante ans après, Jean José Marchand fait alors appel à l'écrivain Dominique de Roux, son cadet de quinze ans - qui n'appartenait pas au monde de la télévision et qu'il n'avait jamais rencontré - comme intervieweur. Éditeur, écrivain, gaulliste bien sûr et ardent polémiste, Dominique de Roux a été le fondateur au début des années 1960 des Cahiers de l'Herne qui ont fait de lui un maître de l'histoire littéraire. Il faut aussi ajouter que cette embauche permettait à Jean José Marchand d'échapper aux règles syndicales qui interdisent qu'un chef de service mène lui-même des entretiens.

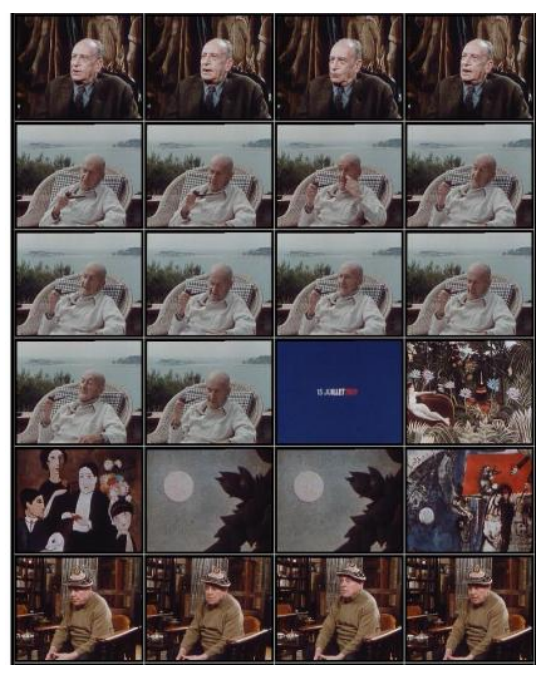

Philippe Soupault, André Salmon, Pierre Mac Orlan dans Apollinaire cinquante ans après, réalisateur : Robert Valey, producteur : Dominique de Roux [Jean José Marchand], 1968 (INA).

\footnotetext{
${ }^{7}$ Participent à cette émission André Salmon, Philippe Soupault, Pierre Mac Orlan, Georges Ribemont Dessaignes, Sonia Delaunay, Roland Dorgelès, André Billy, Romain Weingarten, Claude Bonnefoy, Pierre Schaeffer, Louis Durey, Daniel Henry Kahnweiler, Georges Mathieu, Michel Bernard, Denis Roche, Henri Pichette, Jean-Jacques Brochier, Gabrielle Buffet Picabia.
} 


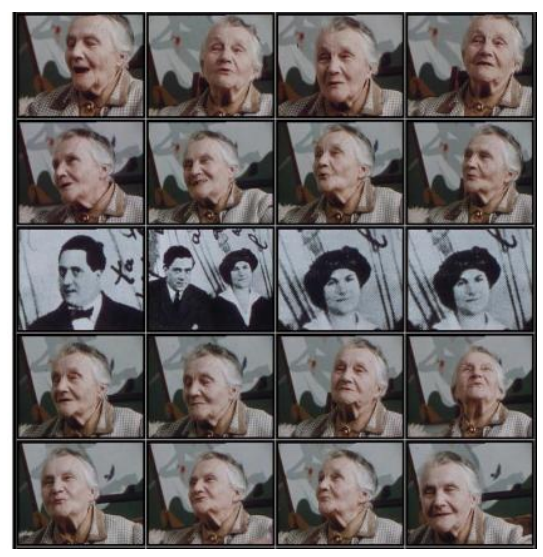

Gabrielle Buffet Picabia dans Apollinaire cinquante ans après, réalisateur : Robert Valey, producteur :

Dominique de Roux [Jean José Marchand], 1968 (INA).

«Ce n'est pas à proprement parler une émission réussie, mais c'est un document formidable », voilà ce que pensait encore récemment Jean José Marchand de son Apollinaire cinquante ans après ${ }^{8}$. Parmi toutes celles interviewées dans ce film, une personnalité l'intrigue plus que les autres. Il s'agit d'André Salmon, poète d'avant-garde durant l'entre-deux-guerres, proche de la collaboration pendant la Seconde Guerre mondiale, chroniqueur permanent de l'art de son temps. Jean José Marchand croit que Salmon aurait beaucoup de choses à dire et compte l'inviter bientôt pour cette fois parler de lui. Mais André Salmon meurt le 13 mars 1969 à l'âge de 88 ans, peu de semaines après la diffusion de son Apollinaire le 6 décembre 1968. Ce fait divers suscite chez Jean José Marchand une forte émotion : tous les témoins de l'art, de la littérature, de la danse et de la musique du $\mathrm{XX}^{\mathrm{e}}$ siècle, encore vivants en cette fin des années 1960 vont bientôt disparaître. Si aucune procédure d'urgence n'est imaginée pour capter leur témoignage, le préjudice intellectuel sera irréparable.

Fort heureusement - et quoiqu'en ait pensé Jean José Marchand quarante ans plus tard l'émission sur Apollinaire marque les esprits. Voir et entendre les témoins de ce qui est toujours considéré comme la grande époque de l'art occidental, le socle sur lequel s'appuient encore aujourd'hui les arts contemporains, a intéressé nombre de spectateurs dont les membres de l'administration de l'ORTF. Jean José Marchand dresse aussitôt une liste de 150 noms qu'il faudrait rapidement contacter et filmer. Cette liste est en quelque sorte le certificat de baptême des Archives du $\mathrm{XX}^{\mathrm{e}}$ siècle qui deviendront bientôt la grande affaire de sa vie : presque quinquagénaire, Jean José Marchand souhaite agir pour durablement baliser le temps qui passe, pour en organiser le souvenir positif, vivant.

\footnotetext{
${ }^{8}$ Cloquet, loc.cit.
} 
La série est inaugurée par une vaste enquête sur le mouvement Dada en cinq émissions d'une heure : Naissance de l'esprit dada, Dada à Zurich, Dada à Berlin, Dada à Paris, Dada à Mantoue ${ }^{9}$ avec entre autres Georges Ribemont-Dessaignes, Gabrielle Buffet Picabia, Juliette Roche, Maximilien Gauthier, Richard Huelsenbeck, Raoul Haussmann, Hans Richter, Marcel Janco, Christian Schad, Philippe Soupault... L'émission est une bonne affaire pour l'ORTF ; elle est vendue et vue dans des pays scandinaves, en Allemagne, en Italie. Ce succès est providentiel. Il permet à Jean José Marchand de se lancer dans ce qui est son véritable projet, à contre courant de tout ce qui se conçoit habituellement dans sa profession : mener des entretiens filmés qui ne s'inscrivent pas dans une programmation longuement planifiée, des entretiens d'abord considérés comme des archives, ainsi que le nom de la série l'indique avec clarté. Tant mieux si quelques-uns trouvent le chemin de la salle de montage puis des écrans de télévision à l'occasion d'un anniversaire, d'une disparition ou d'un créneau horaire à combler ; en ce qui le concerne, Jean José Marchand entend bien préserver son action de tout souci de diffusion immédiate.

Aujourd'hui, les Archives du $\mathrm{XX}^{\mathrm{e}}$ siècle forment une collection rassemblant plus de plus de 150 entretiens dont ceux pour le seul domaine de l'art (outre les noms mentionnés plus haut) réalisés avec Georgette Camille, Marc Chagall, Alfred Courmes, Serge Charchoune, Giorgio de Chirico, André Fougeron, Irène Lagut, Marianne Lam, Mme Marinetti, Édouard Pignon, Boris Taslitsky, Jacques Lipchitz, Madame André Lhote, Joseph Czapski, Marcel Duchamp ou Alice Derain. Captés pour l'essentiel entre 1968 et 1974, ils constituent un gisement exceptionnel d'environ 300000 mètres de pellicules en $16 \mathrm{~mm}$. Mais comment Jean José Marchand a-t-il pu obtenir le renouvellement des budgets pour quelque chose qui passait peu à l'antenne?

\section{Un maître de l'interview}

La réponse à cette question est simple : il fallait être un bon manœuvrier dans les rapports de force internes à la maison ORTF où Jean José Marchand avait ses détracteurs et ses protecteurs. Parmi ces derniers, Pierre Sabbagh (1918-1994), tour à tour journaliste, réalisateur et producteurs d'émissions. Bien connu pour sa fameuse série Au théâtre ce soir qu'il a créée en 1966, Pierre Sabbagh est également celui qui a conçu et présenté le premier journal télédiffusé en France à partir de 1949. Celui-ci sera le plus fidèle et le plus efficace des soutiens à Jean José Marchand et à ses Archives du $\mathrm{XX}^{\mathrm{e}}$ siècle en mettant à sa disposition les moyens nécessaires à son entreprise et en lui

\footnotetext{
${ }^{9}$ Jean José Marchand est épaulé pour les recherches par Yves Poupard-Lieussou. Dans l'équipe, on compte également Suzanne Bujot, l'épouse de Jean José Marchand depuis 1960 et sa collaboratrice dans tous les épisodes et durant toutes les années des Archives du $\mathrm{XX}^{\mathrm{e}}$ siècle.
} 
assurant une entière liberté dans le choix de ses sujets. Mais encore, plus précisément, c'est en regardant le journal télévisé de Pierre Sabbagh que Jean José Marchand élaborera son art et fixera sa manière de mener un entretien filmé, au point d'être considéré comme un maître de l'interview : caméra statique, cadrage frontal ou de troisquarts de la personne interviewée, voix off de l'intervieweur, refus de l'anecdote animée (type promenade dans un jardin...), questionnaire biographique soigneusement préparé, primat accordé au visage et à la voix de façon à ce que le propos de l'invité soit confronté à son expressivité. Il utilise pleinement la « vérité » de la caméra qui guette les émotions, détecte les hésitations, révèle des non-dits, comme en témoigne Philippe Collin, le réalisateur préféré de Jean José Marchand, celui qu'il sollicitait toujours le premier pour un nouveau projet :

Que tout filmeur sache qu'un créateur, agissant ou se racontant par ses gestes, ses regards, son temps de parole, crée sa mise en scène. En se ' contentant' d'enregistrer cette organisation de l'espace et du temps, le paysage à peine mobile de ce visage, en sachant ne pas couper quand l'insignifiance se profile, on met de son côté les chances de transmettre un document d'autant plus rebelle aux manipulations tendancieuses qu'il ne fut pas destiné à promouvoir les mérites du maitre d'œuvre audiovisuel. Cette pratique et cette morale furent celle des Archives $d u X X^{e}$ siècle de Jean José Marchand. ${ }^{10}$

La conception du questionnaire apparaissait fondamentale à Jean José Marchand. Qu'il mène ou non les entretiens, c'est toujours lui qui est l'auteur des questionnaires. Les choses devaient être faites comme dans les « rapports de police », disait-il à Guillaume Louet, « c'est-à-dire disant ' 'untel, il est passé à telle date à Ascona, à telle date il se trouvait à l'hôtel Adlon, à Berlin, à un autre moment on le retrouve à New York', ... » Méthode dont l'originalité était d'être - en apparence - sans originalité : ce questionnaire, établi à partir de la biographie de l'invité, de son œuvre et de sa réception, était envoyé avant le tournage au futur interviewé qui avait ainsi la possibilité de l'amender. À partir de questions simples, le regard, les gestes, l'état d'esprit, la contenance de la personne interviewée, prennent du relief. L'innocuité apparente du questionnaire, sa manière à la fois douce et naturelle de s'introduire dans le parcours d'une vie, pouvaient aussi quelquefois susciter une « libération de la parole » qui alors s'éloignait du parcours minutieusement fléché. En tout état de cause, quel que soit le

\footnotetext{
${ }^{10}$ Philippe Collin, «À propos des Archives du XX $\mathrm{XX}^{\mathrm{e}}$ siècle » dans Abécédaire des films sur l'art moderne et contemporain 1905-1984, Paris, Centre Georges Pompidou / Centre national des arts plastiques, 1985, p. 23
} 
tour que pouvait prendre un entretien, jamais l'intervieweur ne devait se transformer en un commentateur ${ }^{11}$.

À la radio, pense Jean José Marchand, la parole nous attache « à sa dialectique » interne ; on n'entend que la leçon portée par la voix. En revanche, à la télévision « le personnage vous saute à la figure ». Dans un élan qui rappelle le «Ça a été » du Roland Barthes de La Chambre claire, Jean José Marchand loue la télévision pour sa capacité à imposer une présence : « Ils sont là », s'exclame-t-il dans l'entretien qu'il donne à Guillaume Louet, «ils sont là » toutes ces personnalités avec lesquelles il s'est entretenu au nom de ce grand média moderne qui peut livrer au téléspectateur un surplus d'âme. Durant les années 1960 - ne l'oublions pas - la télévision apparaissait encore comme un puissant facteur de démocratie pouvant reprendre à son compte l'idéal réformiste d'élévation culturelle collective. On sait comment cet idéalisme des premières décennies a été battu en brèche par le marché du divertissement de masse dont la domination en France correspond à l'éclatement de l'ORTF en 1974 sous la présidence de Valéry Giscard d'Estaing, homme auquel Jean José Marchand vouait encore hier un mépris souverain. Les hommes politiques - reconnaissait Jean José Marchand - savent souvent bien se servir ou être bien servi par la télévision dont ils mesurent la capacité d'intrusion dans les foyers. Le général de Gaulle, François Mitterrand pour ne pas parler de Jean-Marie Le Pen, avaient un charisme télévisuel donnant à leurs interventions une aura de vérité même lorsqu'ils mentaient. Mais les créateurs, artistes ou écrivains, c'est autre chose : ils hésitent moins à exprimer la vérité - volontairement ou non - «parce que ce sont des gens qui sont tournés vers euxmêmes pense Jean José Marchand, tôt où tard durant l'entretien, «ils se trahissent ». L'éclatement de l'ORTF marque la fin des Archives du XX ${ }^{\mathrm{e}}$ siècle. Jean José Marchand devient alors conseiller de Jean-Charles Edeline à la Société française de production (SFP), société de droits privée à capitaux publics née de cet éclatement. Il y restera jusqu'en 1981 et l'arrivée des socialistes au pouvoir. Jean-Charles Edeline est un directeur bienveillant, mais il ne peut allouer à Jean José Marchand aucun budget spécifique. Au chaud pendant huit ans, jusqu'à la retraite, dans cette sorte de placard, il continue à monter certaines archives grâce aux outils de la SFP, mais ne tourne rien si ce n'est un Chagall. En 1974, une Association des amis des Archives du XX ${ }^{\mathrm{e}}$ siècle est créée afin de donner au projet une assise juridique permettant la diversification des financements, afin également de trouver de nouveaux partenaires prêts à exploiter les archives sous des formes originales, une banque de données audiovisuelles, par

\footnotetext{
${ }^{11}$ Le plus souvent, le tournage de l'entretien s'étalait sur deux jours et se déroulait de préférence au domicile de l'invité. Ces deux jours de tournage sont en général précédés de huit jours de préparation. L'étape du montage dure environ quatre semaines.
} 
exemple, au service des musées, des universités, des maisons de la culture ou encore des ambassades et des consulats de France à l'étranger. Cette recherche ne sera pas totalement vaine puisque le Centre Pompidou, qui en 1975 n'a pas encore vu le jour, intègre les Archives du $\mathrm{XX}^{\mathrm{e}}$ siècle dans son projet. Mais cette association ne perdure que trois ans. Le coût des « frais externes »-c'est-à-dire notamment les salaires et les déplacements des équipes de tournage - apparaissent rapidement excessifs au Centre Pompidou au moment où la captation vidéo, légère et bon marché, prend son envol ${ }^{12}$. Mais cette association permet tout de même l'enregistrement d'une dizaine d'entretiens avec Alfred Courmes, Jean Dewasne, André Fougeron, Jacques Hérold, Marcel Jean, Matta, Édouard Pignon, Gérard Singer, Boris Taslitzky, Patrick Waldberg.

Cette année-là cependant, le véritable espoir de Jean José Marchand réside dans une percée technologique annoncée, dans la mise au point des premiers magnétoscopes, une invention qui permettra - il en est certain ${ }^{13}$ - de se lancer à l'horizon des années $1980^{14}$ dans une véritable exploitation des archives qui pourront alors être diffusées à une heure incertaine de la nuit, enregistrées et regardées en différé, au moment qui semblera le plus propice à chacun. En attendant, on essaie de donner aux entretiens un public sinon de téléspectateurs, au moins de lecteurs à travers le lancement d'une collection «Archives du XX ${ }^{\mathrm{e}}$ siècle » aux Éditions Jean-Michel Place. Deux ouvrages reproduisant intégralement le témoignage des écrivains verront le jour : un Henry de Montherlant interrogé par Jean José Marchand, et un Gabriel Marcel interrogé par Pierre Boutang ${ }^{15}$.

\footnotetext{
${ }^{12}$ Lettre du département audiovisuel du Centre Pompidou à Philippe Collin, 5 février 1979.

${ }^{13}$ Note datée du 4 juillet 1975.

${ }^{14}$ Les Archives du $\mathrm{XX}^{\mathrm{e}}$ siècle seront brièvement prolongées durant les années 1980 par la série Mémoire du $\mathrm{XX}^{\mathrm{e}}$ siècle, animée par Pierre-André Boutang. Mais ce sont tous des entretiens destinés dès le départ à être montées, loin du risque de l'archive.

${ }^{15}$ Le Montherlant a été publié par Jean-Michel Place en 1974 et le Gabriel Marcel interrogé par Pierre Boutang suivi de Position et approches concrètes du mystère ontologique en 1977. Il faut ajouter à cette courte liste un Paul Morand. Entretien avec Jean José Marchand, publié par la Table ronde en 1990, puis en 2001. Une édition des écrits de Jean José Marchand rassemblant ses textes de critique littéraire et artistique est en préparation sous la responsabilité de Guillaume Louet. Pour défendre ce projet ainsi que la promotion des Archives, l'Association des amis des Archives du XX $X^{\mathrm{e}}$ siècle (loi de 1901) fondée en 1976 par Jean José Marchand a récemment été réactivée : Philippe Collin en est le président, Suzanne Marchand la trésorière et Guillaume Louet le secrétaire général. À noter qu'il existe une version dvd des entretiens avec Claude Lévi-Strauss aux éditions Montparnasse en 2006, et que d'autres éditions de dvd sont à l'étude.
}

Le film sur l'art, entre histoire de l'art et documentaire de création Université de Lausanne, 14-16 avril 2011

À paraître aux Presses universitaires de Rennes 
Le bilan des Archives du XX ${ }^{\mathrm{e}}$ siècle de Jean José Marchand - je l'ai dit plus haut - ce sont plus de 150 interviews réalisés. Mais ce sont aussi une cinquantaine de projets non concrétisés pour cause de « fin de l'ORTF ». Il faut par ailleurs évoquer les refus : Aragon par exemple qui décline la demande de Jean José Marchand de participer à l'émission Dada sous le prétexte que son concepteur travaillait pour « la télévision du pouvoir », ou Georges Balanchine qui lui fait répondre par Barbara Hogan, son assistante au New York City Ballet en 1976 que l'avenir et ses archives lui importent peu dans la mesure où cet avenir se fera sans lui ${ }^{16}$. Parmi les grands absents des Archives du $\mathrm{XX}^{\mathrm{e}}$ siècle, on peut citer André Malraux, dont la geste trop connue ne nécessite aucun accessoire supplémentaire ; il y a aussi Jean-Paul Sartre parce Jean José Marchand était persuadé d'essuyer un refus. Michel Leiris, René Char, Julien Gracq, de leur côté, ne voulaient pas être filmés ; un reste d'animisme les habitait, pense Jean José Marchand, ils craignaient sans doute qu'une partie de leur âme passe sur la pellicule. Michel Foucault a répondu «plus tard », ce qui en général signifie « jamais ». Le peintre Joseph Sima, lui, meurt la veille de l'enregistrement le 24 juillet 1971. Le plus long entretien a été celui mené avec Jorge Luis Borgès : neuf heures en quatre jours. Le plus court a été celui d'Ezra Pound qui, une fois devant la caméra, a refusé net de parler. Mais ce silence dit quelque chose et les images laissées par cet entretien paradoxal celles d'un vieillard immensément beau plongé dans une rêverie sans fin - sont parmi les plus puissantes qu'aient laissées les Archives du $\mathrm{XX}^{\mathrm{e}}$ siècle. Elles «figurent » ce qui se trouve au cœur du projet de Jean José Marchand.

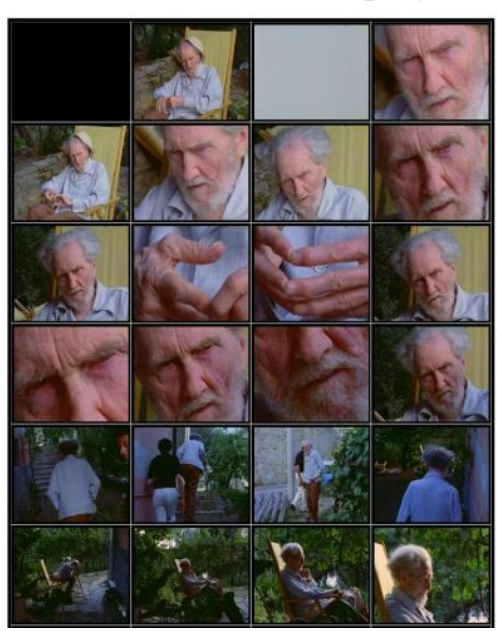

Ezra Pound, rushes, série Archives du XXe siècle, (SFP-INA)

\footnotetext{
${ }^{16}$ « He replys that he is not interrested in the future, as he will not be here. » (Barbara Horgan à Jean José Marchand, 19 janvier 1976).
} 